\title{
Influencia de la Densidad Seca y el Contenido de Agua de Compactación en las Curvas Exploratorias de un Suelo Residual Derivado de Ceniza Volcánica
}

\section{Influence of the Dry Density and Compaction Water Content on the Scanning Drying Curve of a Residual Soil Derived from Volcanic Ash}

DOI: http://dx.doi.org/10.17981/ingecuc.13.1.2017.02

Artículo de investigación. Fecha de recepción: 6 de septiembre de 2016. Fecha de aceptación: 17 de diciembre de 2016

\author{
Angélica Marcela Orjuela Garzón \\ Universidad Nacional de Colombia. Bogotá, (Colombia) \\ amorjuelag@unal.edu.co \\ Julio Esteban Colmenares Montañez \\ Universidad Nacional de Colombia. Bogotá, (Colombia) \\ jecolmenaresm@unal.edu.co \\ Livaniel Viveros Rosero \\ Universidad Nacional de Colombia. Bogotá, (Colombia) \\ lviverosr@unal.edu.co
}

Para citar este artículo:

A. M. Orjuela Garzón, J. E. Colmenares Montañez y L. Viveros Rosero, "Influencia de la densidad seca y el contenido de agua de compactación en las curvas exploratorias de un suelo residual derivado de ceniza volcánica," INGE CUC, vol. 13, no. 1, pp. 19-31, 2017.

\section{Resumen}

Introducción- Una curva exploratoria de humedecimiento o secado relaciona los resultados de mediciones sucesivas del contenido de agua y la succión de un suelo originalmente en equilibrio que parte de una condición inicial de saturación parcial y sigue un proceso de humedecimiento o secado.

Objetivo- Estudiar la influencia de la densidad seca y el contenido de agua de compactación en la curva exploratoria de un suelo residual derivado de ceniza volcánica, el cual se sometió a un proceso de secado expuesto al ambiente.

Metodología- El trabajo experimental inició con la determinación de la curva de compactación del material estudiado. Con base en ello, se seleccionaron seis puntos dentro de la curva que permitieron estudiar la relación existente entre la densidad seca y el contenido de agua de compactación con la succión a través de la compactación estática de muestras del suelo estudiado, usando para tal fin curvas exploratorias de secado a través de la técnica del papel de filtro.

Resultados- La densidad seca inicial del material no influye en las trayectorias que describen las curvas exploratorias para valores similares de succión. El contenido de agua de compactación influye en la capacidad de almacenamiento del suelo estudiado, encontrándose que, para un mismo valor de densidad, muestras compactadas del lado húmedo del contenido de agua óptimo presentan mayores valores de succión que muestras compactadas del lado seco.

Conclusiones- La capacidad de almacenamiento de agua del suelo estudiado es independiente de la densidad seca inicial del material. La capacidad de almacenamiento de agua del suelo estudiado está influenciada por el contenido de agua de compactación, encontrándose que muestras compactadas del lado húmedo del contenido de agua óptimo presentan mayores valores de succión que muestras compactadas del lado seco.

Palabras clave- Curva de retención de agua, curva exploratoria, succión, compactación, conductividad hidráulica
Abstract

Introduction- Scanning wetting or drying curves show the relation between successive measurements of water content and suction of an originally partly saturated soil which follows a drying or wetting process.

Objective- To study the influence of dry density and compaction water content into a scanning drying curve of a residual soil derived from volcanic ash, which was subjected to a drying process exposed to the environment.

Methodology- The experimental work started with the determination of compaction curve of the studied material. Based on the curve, six points were selected within the curve. It allowed studying the existent relation between dry density and compaction water content with suction through the static compaction of the studied soil using for that aim, scanning drying curves obtained from the filter paper technique.

Results- Initial dry density of the material does not influence into the paths that describe the scanning drying curves to similar suction values.

The compaction water content influences into the storage capacity of the studied soil. It was found that for the same density value, samples compacted to the wet side of optimum water content, show higher suction values than samples compacted of the dry side of optimum water content.

Conclusions- Water storage capacity of the studied soil is independent of the initial dry density of the material.

Water storage capacity of the studied soil is influenced by the compaction water content. It was found that samples compacted to the wet side of the optimum water content show higher suction values than samples compacted of the dry side of optimum water content.

Keywords- Soil water retention curve, Scanning curve, suction, compaction, hydraulic conductivity. 


\section{Introducción}

La zona occidental del cono sur del continente americano se destaca por la presencia de grandes complejos volcánicos a lo largo de la Cordillera de Los Andes. Debido a esta condición tectónica, en toda la extensión de la cordillera Central colombiana se presentan tres grandes sistemas volcánicos: el sistema Ruiz-Tolima, el sistema Huila-Puracé y el sistema Galeras-Azufral. La actividad de estos sistemas volcánicos ha generado la acumulación de grandes depósitos de cenizas volcánicas que por procesos de meteorización han permitido la formación de suelos residuales.

Métodos de compactación comúnmente usados en ingeniería han sido aplicados sobre este tipo de suelos sin una base experimental adecuada que permita determinar que su comportamiento se ajusta a los métodos convencionales de construcción, lo que ha llevado a la generación de problemas geotécnicos. Se ha encontrado, por ejemplo, que los suelos residuales originados a partir de las cenizas volcánicas presentan cambios en su comportamiento producto de la desecación del material. De acuerdo con [1], dentro de los problemas geotécnicos que comúnmente se encuentran en este tipo de suelos están: estabilidad de taludes, alta sensibilidad, agrietamiento y problemas durante su manejo como material de construcción.

La succión es un componente que contribuye a la resistencia del suelo y a la estabilidad natural de taludes de gran altura e inclinación, comúnmente observados en suelos derivados de ceniza volcánica [1]. La relación succión-contenido de agua, expresada a través de curvas exploratorias, ha constituido un elemento clave en el desarrollo de la mecánica de suelos parcialmente saturados.

Una curva exploratoria de humedecimiento o secado relaciona los resultados de mediciones sucesivas de contenido de agua y succión de un suelo, originalmente en equilibrio, que parte de una condición inicial de saturación parcial y sigue un proceso de humedecimiento o secado. Estas curvas se desarrollan dentro de las fronteras definidas por las curvas de retención de agua del suelo originalmente saturado, que sigue un proceso de secado, y la del suelo originalmente seco, que sigue un proceso de humedecimiento. De acuerdo con [2](ii, aplicaciones de esta relación, contenido de agua-succión, han sido útiles para la estimación de funciones de propiedades como la resistencia al corte, la conductividad hidráulica y el comportamiento volumétrico de los suelos parcialmente saturados. Así, por ejemplo, la medición de la relación succión-contenido de agua, determinada a través de las curvas exploratorias, puede ser utilizada para estimar las propiedades mecánicas de un suelo parcialmente saturado.

Factores como las condiciones climáticas imperantes afectan el comportamiento mecánico del suelo, lo que da lugar a problemas geotécnicos asociados a fallas de taludes o deslizamientos de tierra. Periodos secos generan la evaporación del agua de los poros del suelo dando lugar a la desecación, la formación de grietas y el incremento de la succión. Por el contrario, periodos de fuertes precipitaciones generan fallas de taludes asociadas a la pérdida de succión y reducción en la resistencia al corte de los materiales, así como el incremento en la infiltración del agua.

Considerando la susceptibilidad de esta clase de suelos a procesos de secado, a continuación, se presenta un análisis de la influencia de la densidad seca y el contenido de agua de compactación en la curva exploratoria de un suelo residual derivado de ceniza volcánica, el cual se sometió a un proceso de secado expuesto al ambiente. Se compararon curvas exploratorias para densidades secas de $0,95 \mathrm{~g} / \mathrm{cm}^{3}$ y $1,15 \mathrm{~g} /$ $\mathrm{cm}^{3}$ con contenidos de agua del lado seco del óptimo, del lado húmedo del óptimo y en la condición óptima. Los resultados experimentales para los contenidos de agua analizados indican que la capacidad de almacenamiento de agua del suelo estudiado es independiente de la densidad seca inicial del material, ya que las curvas exploratorias compactadas bajo diferentes densidades secas presentan comportamientos similares. Por el contrario, la capacidad de almacenamiento del suelo estudiado está influenciada por el contenido de agua de compactación, encontrándose que muestras compactadas del lado húmedo del contenido de agua óptimo presentan mayores valores de succión que muestras compactadas del lado seco.

Se comparó además una curva exploratoria compactada a una densidad seca de $0,95 \mathrm{~g} / \mathrm{cm}^{3}$ con un contenido de agua del lado húmedo del óptimo (casi saturado) con la curva exploratoria obtenida por [3], quien realizó pruebas al mismo material conservando el contenido natural de agua para la compactación encontrándose que los procesos de secado modificaron algunas de las propiedades del suelo.

\section{Marco Metodológico}

El trabajo experimental se inició con la compactación dinámica del material buscando obtener la curva que relaciona el contenido de agua con la densidad seca y a partir de la cual se determinó la densidad seca máxima y el contenido de agua óptimo del material estudiado. Con base en ello, se seleccionaron seis puntos dentro de la curva que permitieron estudiar la relación existente entre la densidad seca y el contenido de agua de compactación con la succión a través de la compactación estática de muestras de suelo derivado de ceniza volcánica, al cual previamente se le habían determinado sus principales características.

A partir de los seis puntos seleccionados, con características de compactación diferentes (densidad seca y contenido de agua), se elaboraron las curvas exploratorias de secado. La succión se determinó mediante la técnica del papel de filtro usando la ecuación de calibración de Chandler y Montgomery-Smith [4]. 
Con base en la información recolectada que permitió dar forma a las curvas exploratorias de secado, se ejecutó un ajuste matemático para cada una de las curvas (en este caso, el de Fredlund y Xing [5]). Luego, se estudió la relación existente entre la densidad seca y la succión a través de la influencia de la densidad seca inicial de compactación sobre las curvas exploratorias de secado, para lo cual se agruparon las curvas exploratorias en tres pares, donde cada par tenía como característica que estaba compactado bajo el mismo contenido de agua pero a densidades secas diferentes. Posteriormente, mediante un modelo teórico presentado por [6], se determinó la distribución del tamaño de poros de cada curva con el objetivo de buscar una relación entre el tamaño de poro con el comportamiento de la curva exploratoria.

Se estudió, además, la relación existente entre el contenido de agua de compactación y la succión a través de la influencia del contenido de agua de compactación sobre la curva exploratoria de secado, para lo cual se agruparon las curvas obtenidas para cada una de las seis características de compactación en grupos de tres, donde cada grupo estaría compactado bajo la misma densidad seca pero con diferente contenido de agua.

Finalmente, se realizó una comparación de la información recolectada con ensayos ejecutados al mismo material en condición natural con el objetivo de estudiar los cambios de sus características físicas al ser secado y remoldeado.

\section{III.Marco Conceptual}

\section{A. Suelos residuales y parcialmente saturados}

Los suelos residuales derivados de ceniza volcánica se generan a partir de procesos de alteración física y química de depósitos de ceniza volcánica alojados alrededor de grandes complejos volcánicos. Estos procesos transforman los minerales, la forma y tamaño de las partículas, la fábrica y la porosidad [7]-[8].

Los suelos derivados de ceniza volcánica, también llamados por la edafología como andosoles o andisoles [9], se encuentran localizados principalmente en países tropicales y subtropicales y están formados por vidrio volcánico. El comportamiento de estos suelos residuales tropicales, de origen volcánico, es fuertemente influenciado por su composición mineralógica, en donde se destaca la presencia de dos minerales arcillosos: la alófana (frecuentemente asociada a otro mineral llamado imogolita) y la haloisita [9].

La alófana, la imogolita y la haloisita, derivados de la meteorización de la ceniza volcánica, poseen propiedades, como el tamaño, la forma o la carga eléctrica, que los distinguen de los minerales comúnmente encontrados en suelos sedimentarios [1]. Estos minerales influyen, entre otros aspectos, sobre los resultados de los límites de Atterberg y no permiten identificar la plasticidad de este tipo de suelo [3].
En Colombia, los suelos residuales han sido usados como material de construcción (p.e. terraplenes, para autopistas, vías férreas y pistas de aterrizaje). La referencia [2](ii muestra que, bajo una condición de saturación parcial, las presiones intersticiales son negativas y, por lo tanto, las propiedades del suelo ya no son definidas como valores únicos, o como parámetros del suelo, sino como funciones de propiedad que dependen del estado de esfuerzos del suelo y, en particular, de la presión negativa cuantificada como succión para describir su comportamiento.

La succión matricial está relacionada con la presencia de meniscos en los poros del suelo que generan una fuerza adicional de unión en los contactos entre granos debido a la tensión superficial del agua que ocupa parcialmente los poros del suelo [10]. Por otra parte, la succión osmótica se relaciona con la presencia de sales disueltas en el agua de poros.

\section{B. Curvas exploratorias de medición de succión}

El contenido de agua del suelo, que a su vez condiciona la energía del agua del mismo, influye fuertemente en su comportamiento mecánico. Una relación muy utilizada para caracterizar y estudiar el comportamiento de los suelos, en condición de saturación parcial, es la curva de retención de agua, que relaciona el contenido de agua y la succión.

La grafica de los resultados de mediciones sucesivas de contenido de agua y succión, en procesos de secado o humedecimiento, conforma una curva exploratoria de secado (Scanning Drying Curve-SDC) o una curva exploratoria de humedecimiento (Scanning Wetting Curve -SWC), que se mueve entre las fronteras que representan las curvas de secado (Primary Drying Curve-PDC) y humedecimiento (Primary Wetting Curve-PWC) iniciales, las cuales corresponden a una muestra saturada que ha sido totalmente secada y a una muestra totalmente seca que ha sido completamente humedecida, respectivamente. En la fig. 1 se aprecia en forma esquemática el fenómeno de la histéresis que se presenta en un suelo típico con esqueleto mineral rígido, es decir, que no presenta cambios de volumen durante el proceso.

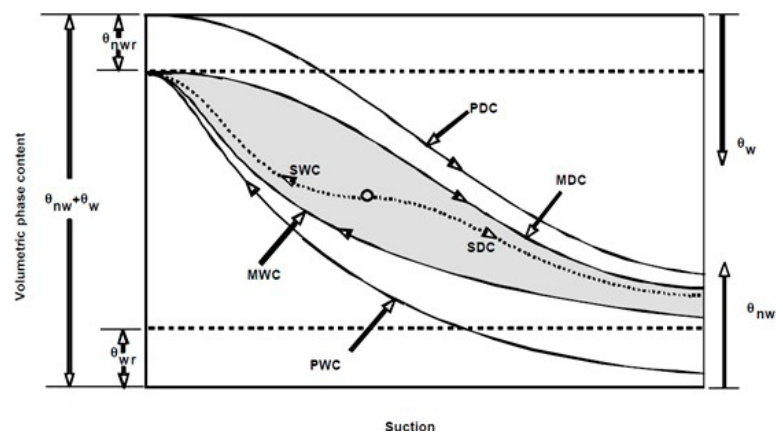

Fig. 1. Representación esquemática del fenómeno de histéresis en un suelo con esqueleto mineral rígido. Fuente: [11] 
Las condiciones y procesos de compactación son factores que inciden en la fábrica del suelo. Estos elementos son esenciales en el conocimiento de las características de retención de agua en cada estado de compactación. La succión del agua asociada a cada estado de compactación sirve como marco para entender el comportamiento del suelo. Diversos autores han mostrado las líneas de iso-succión en suelos compactados sobre las curvas en el diagrama de compactación. La referencia [12] sugiere que las curvas de iso-succión de un material limoso de baja plasticidad son verticales en el diagrama de Proctor cuando el suelo es compactado a contenidos de agua del lado seco del óptimo y que el comportamiento de las líneas de iso-succión tiende a ser paralelo a las líneas de igual grado de saturación cuando se compacta a contenidos de agua mayores. Para [12], la diferencia en el comportamiento está definida principalmente por el valor del contenido óptimo de agua; por lo cual, para el lado seco del óptimo se presenta la tendencia aproximadamente vertical y para el lado húmedo del optimo se presenta la tendencia a seguir las líneas de saturación constante.

\section{Fábrica}

El tamaño, la forma y la distribución de los espacios de poros comprenden un elemento fundamental en el estudio de la fábrica del suelo y juegan un papel importante en el gobierno del comportamiento ingenieril de la masa de suelo, ya que permiten evaluar las relaciones entre el tamaño de poros, la distribución del tamaño de poros y la capilaridad en un suelo no saturado, elementos que proporcionan una información valiosa en la predicción de la compresibilidad o la permeabilidad [6].

Las medidas de succión y contenido volumétrico de agua permiten determinar de manera teórica el volumen y distribución de tamaño de poros. El volumen de poros, el radio de poros promedio, el espesor de la película de agua adsorbida en los sólidos del suelo y la relación de volumen de poros y área superficial permiten conceptualizar la geometría de los poros del suelo con base en la teoría de la capilaridad.

Debido a que los procedimientos computacionales para estimar la distribución del tamaño de poros envuelven integraciones numéricas, es importante definir esas cantidades en forma incremental como función de la humedad relativa o la succión matricial. Cada uno puede ser cuantificado en cantidades incrementales a lo largo de la curva de retención de agua del suelo en estudio [6].

\section{Susceptibilidad al secado y remoldeo}

Los suelos derivados de ceniza volcánica presentan cambios fuertes e irreversibles debidos a procesos de secado. Este comportamiento se debe posiblemente a la pérdida de agua retenida por los minerales arcillosos que caracterizan a este tipo de suelos. El suelo producto del secado no tiene la capacidad de retención de agua inicial y no permite formar una estructura parecida a la desarrollada con un suelo natural [1].
Se ha demostrado que los materiales derivados de ceniza volcánica, compuestos por alófana y haloisita, pierden sus características de plasticidad cuando son secados al aire o en el horno [13].

La alta plasticidad que estos suelos presentan en condición natural se reduce cuando el suelo es expuesto a secado al aire o al horno. Los cambios se hacen más severos para suelos con una plasticidad natural alta y a medida que ocurre mayor pérdida de agua previa al ensayo [1].

\section{Material de Estudio}

\section{A. Procedencia del material y tipo de suelo estudiado}

El material estudiado proviene de las formaciones superficiales de un suelo derivado de ceniza volcánica de un sector ubicado en el municipio de Palestina, en el departamento de Caldas, Colombia, el cual tiene su origen en la alteración física y química de los depósitos de ceniza volcánica producidos por la actividad explosiva moderada del volcán Nevado del Ruiz [3]. Para el caso de este estudio, se usó el material a granel, el cual se encontraba en estado inicialmente seco al aire.

En el área de influencia se identificaron formaciones superficiales entre las cuales se encuentran los depósitos de caída piroclástica, suelos residuales originados por la meteorización de rocas metamórficas y depósitos de ladera producto de antiguos movimientos en masa.

\section{B. Caracterización básica}

Para la caracterización básica del material estudiado, se siguieron los procedimientos establecidos por la American Society for Testing and Materials (ASTM). Dado que se trata de suelos de composición especial, se tuvieron en cuenta las recomendaciones dadas por [3] para este tipo de suelo residual. La Tabla 1 muestra los resultados obtenidos:

Tabla 1. Características generales del suelo estudiado.

\begin{tabular}{|c|c|}
\hline \multicolumn{2}{|c|}{ Características del suelo estudiado } \\
\hline Descripción visual & $\begin{array}{c}\text { Arena limosa fina, } \\
\text { sin plasticidad }\end{array}$ \\
\hline Color & Café claro \\
\hline Contenido de agua (\%) & 7,4 \\
\hline Gravedad específica & 2,67 \\
\hline Límite líquido (\%) & $\mathrm{NL}$ \\
\hline Límite plástico (\%) & $\mathrm{NP}$ \\
\hline Partículas de tamaño arena (\%) & 58,66 \\
\hline Partículas de tamaño limo (\%) & 36,71 \\
\hline Partículas de tamaño arcilla (\%) & 4,63 \\
\hline Clasificación USCS & $\mathrm{SP}$ \\
\hline
\end{tabular}

Fuente: Autores 


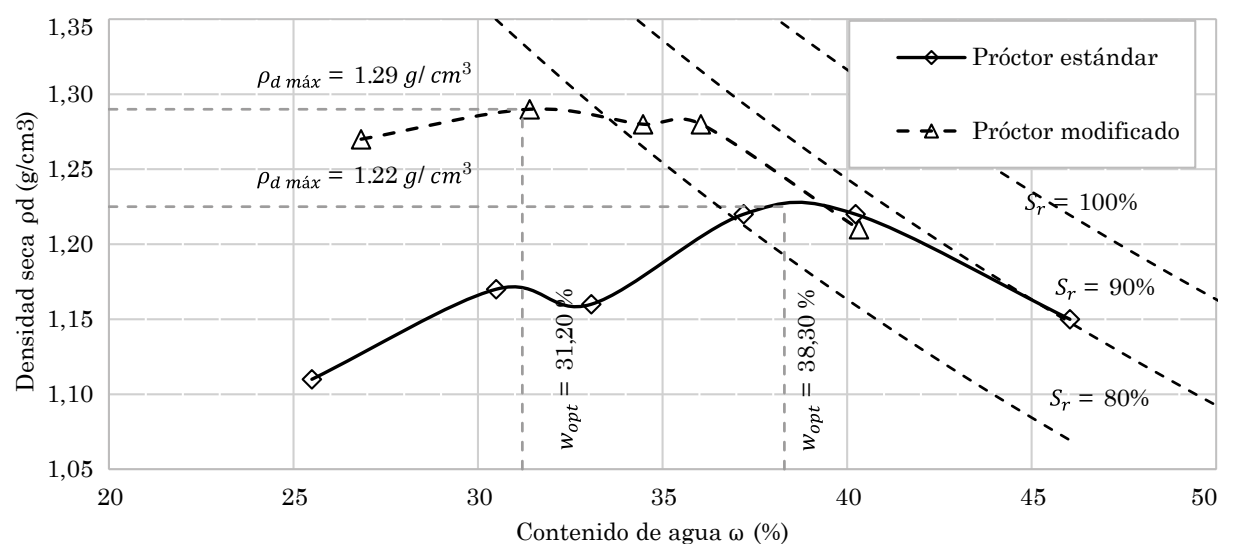

Fig. 2. Curvas de compactación. Fuente: Autores

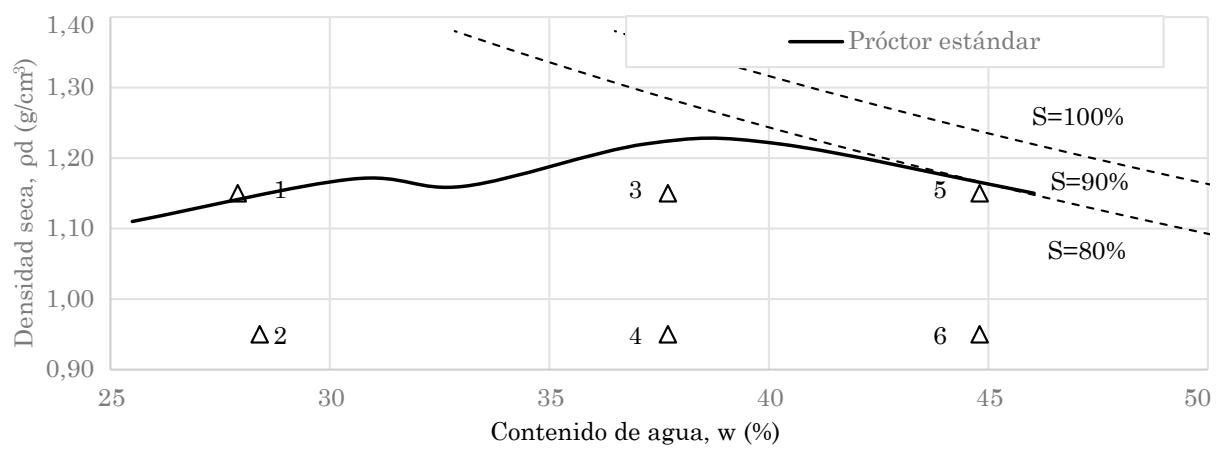

Fig. 3. Características de compactación de seis muestras usadas para el estudio experimental de las curvas exploratorias. Fuente: Autores

\section{Caracterización química y mineralógica}

Se llevaron a cabo pruebas para la determinación de la composición química y mineralógica del suelo estudiado. En el primer caso, se utilizó un espectrómetro de fluorescencia de rayos X para analizar la concentración de elementos químicos de la muestra en estudio. En los resultados se encontró que el suelo se caracteriza por la abundancia en el contenido de óxidos: óxido de sílice $\left(\mathrm{SiO}_{2}\right)$, óxido de aluminio $\left(\mathrm{AL}_{2} \mathrm{O}_{3}\right)$ y óxido de hierro $\left(\mathrm{Fe}_{2} \mathrm{O}_{3}\right)$, principalmente. El comportamiento mecánico de suelos residuales tropicales de origen volcánico es fuertemente influenciado por la presencia de minerales silicatos (como la alófana y la haloisita), y pueden contener minerales no silicatos o minerales de óxido como el óxido de aluminio y el óxido de hierro [9].

En el análisis de la composición mineralógica, se utilizó la técnica de difracción por rayos X. En los resultados se encontró presencia de cuarzo, alumino-silicatos hidratados amorfos (alófana) con una estructura no cristalina y hematites. Se realizó además un análisis petrográfico de sección delgada para uno de los bloques recuperados con el fin de determinar la composición y las estructuras internas del material. De manera general, los resultados muestran que el material evaluado es un suelo residual compuesto de líticos de rocas volcánicas, cristales de hornablendas y plagioclasas intermedias. Por lo tanto, el material es de naturaleza clástica y presenta materiales accesorios como biotita, vidrio volcánico, plagioclasas, hornablenda y piroxeno.

\section{Programa Experimental}

\section{A. Procesos de compactación dinámica y estática}

Las curvas de compactación realizadas de acuerdo con la norma ASTM D 698 [14] y ASTM D 1557 [15], se muestran en la figura 2, y las características más importantes de los ensayos de compactación se muestran en la Tabla 2 .

Tabla 2. Características de compactación del suelo analizado.

\begin{tabular}{|c|c|c|c|}
\hline \multicolumn{2}{|c|}{$\begin{array}{c}\text { Método de } \\
\text { compactación normal }\end{array}$} & \multicolumn{2}{c|}{$\begin{array}{c}\text { Método de } \\
\text { compactación modificado }\end{array}$} \\
\hline $\begin{array}{c}\text { Densidad } \\
\text { seca máxima } \\
(\mathrm{g} / \mathrm{cm} 3)\end{array}$ & $\begin{array}{c}\text { Contenido } \\
\text { de agua } \\
\text { óptimo (\%) }\end{array}$ & $\begin{array}{c}\text { Densidad } \\
\text { seca máxima } \\
(\mathrm{g} / \mathrm{cm} 3)\end{array}$ & $\begin{array}{c}\text { Contenido } \\
\text { de agua } \\
\text { óptimo (\%) }\end{array}$ \\
\hline 1.22 & 38.30 & 1.29 & 31.20 \\
\hline
\end{tabular}

Fuente: Autores 


\section{B. Preparación de las muestras}

Para garantizar la uniformidad de las muestras utilizadas en este estudio, se utilizó la compactación estática por esfuerzo pico variable con deformación constante, la cual permite una aplicación gradual de la fuerza a una masa de suelo suelta hasta que se consigue un determinado espesor final [16].

Se compactaron seis especímenes, de acuerdo con el procedimiento anteriormente descrito, con el objetivo de analizar la influencia de la densidad seca y el contenido de agua de compactación iniciales del suelo sobre las curvas exploratorias. La Tabla 2 presenta los valores de densidad seca y contenido de agua bajo los cuales se prepararon las muestras. El contenido inicial de agua usado representa: la condición seca (muestras 1 y 2), la condición óptima encontrada en el ensayo de Proctor estándar (muestras 3 y 4) y la condición húmeda (muestras 5 y 6) del material estudiado. Los resultados se analizaron a través de la comparación del contenido gravimétrico de agua con la succión matricial.

Las curvas de secado analizadas no parten de una saturación de $100 \%$, ya que las muestras fueron compactadas bajo contenidos de agua por debajo de la saturación. Dado que en ninguno de los casos el suelo parte de una succión correspondiente a $0 \mathrm{kPa}$, se trata entonces de curvas exploratorias (Scanning Drying Curves - $S D C$ ).

\section{Medición de succión por el método del papel de filtro}

El papel de filtro es un método comúnmente usado para la medición de la succión. El contenido de agua del papel de filtro en contacto directo con la muestra de suelo se asocia con la succión matricial del agua en el suelo y su rango de medición es muy amplio. Existe otro método en el que no se presenta contacto entre la muestra de suelo y el papel de filtro con rangos de medición entre los $1000 \mathrm{kPa}$ y los $500000 \mathrm{kPa}$. Ambas técnicas, la de contacto y no contacto del papel filtro, proporcionan un estimativo de la succión del suelo indirectamente mediante la medición del agua transferida desde un espécimen de suelo no saturado a un papel de filtro inicialmente seco [6].
Las mediciones de succión matricial se realizaron mediante la técnica del papel de filtro. El procedimiento consistió en colocar dos papeles de filtro inicialmente secos sobre las dos caras de la muestra y un ensamble de dos discos de acrílico cubriendo los papeles de filtro. La muestra de suelo y el papel de filtro se mantuvieron en contacto mediante la presión suave ejercida por los discos de acrílico. Otros detalles del procedimiento son descritos por [17]. Cumplido el periodo de equilibrio, se midió el contenido de agua del papel de filtro en contacto con la muestra y se determinó el valor de la succión mediante una ecuación de calibración. Se utilizó papel de filtro Whatman No. 42 y el valor de la succión se determinó a través de la ecuación de calibración propuesta por [4].

\section{Ajuste de la curva exploratoria}

Las técnicas experimentales para la medición de la curva de retención de agua permiten determinar un número limitado de datos. Sin embargo, para relacionar la succión y el contenido de agua con parámetros geotécnicos como cambios volumétricos, resistencia al corte o conductividad hidráulica, se hace necesaria la implementación de modelos matemáticos que permitan describir la forma de la curva de retención de agua.

Se han propuesto numerosos modelos matemáticos de aproximación que permiten describir la forma de la curva de retención de agua. La mayoría de estos modelos se basan en la utilización de parámetros fijos relacionados con el contenido de agua o la succión en condiciones específicas (saturación, saturación residual, valor de entrada de aire) y dos o más constantes empíricas o semiempíricas de ajuste, que sirven para capturar la forma general de la curva entre estos puntos fijos [6].

A partir del modelo propuesto por Fredlund y Xing en 1994 [5], las curvas exploratorias obtenidas para diferentes valores de contenido gravimétrico de agua y succión fueron expresadas en forma matemática de acuerdo con la ecuación (1).

$$
w=w_{s}\left[1-\frac{\operatorname{Ln}\left(1+\frac{\psi}{\psi_{r}}\right)}{\operatorname{Ln}\left(1+\frac{10^{6}}{\psi_{r}}\right)}\right]\left[\frac{1}{\operatorname{Ln}\left[e+\left(\frac{\psi}{a}\right)^{n}\right]}\right]^{m}
$$

Tabla 3. Parámetros ajustados de las curvas exploratorias de acuerdo con el modelo de Fredlund y Xing (1994)

\begin{tabular}{|c|c|c|c|c|c|c|}
\hline \multirow{2}{*}{ Parámetro } & \multicolumn{5}{|c|}{ Muestras } \\
\cline { 2 - 8 } & M1 & M2 & M3 & M4 & M5 & M6 \\
\hline Contenido de agua de saturación $(\%)$ & 49,46 & 67,77 & 49,46 & 67,77 & 49,46 & 67,77 \\
\hline Succión residual estimada $(\mathrm{kPa})$ & 16509 & 13292 & 23610 & 13208 & 3689 & 4458 \\
\hline Parámetro de ajuste $a(\mathrm{kPa})$ & 24,78 & 1,64 & 31,23 & 2,24 & 15,84 & 4,44 \\
\hline Parámetro de ajuste $n$ & 0,37 & 0,53 & 0,46 & 0,43 & 0,81 & 0,46 \\
\hline Parámetro de ajuste $m$ & 1,64 & 1,11 & 1,49 & 1,36 & 0,87 & 1,33 \\
\hline
\end{tabular}

Fuente: [5]. 
Donde es el contenido gravimétrico de agua a cualquier valor de succión, es la succión en el suelo en $\mathrm{kPa}$, es la succión estimada en la condición residual, corresponde al contenido gravimétrico de agua del suelo en estado de saturación, es la base del logaritmo natural, es un parámetro del suelo relacionado al valor de entrada de aire, es un parámetro relacionado con la distribución del tamaño de poros y es un parámetro relacionado con la simetría de la curva característica. En la Tabla 3 se presentan los parámetros del modelo matemático propuesto por [5] y utilizado en el presente artículo para describir el comportamiento de las curvas exploratorias de las muestras estudiadas.

\section{Resultados y Discusión}

\section{A. Influencia de la densidad seca inicial}

La forma de las curvas exploratorias sugiere que, a pesar de que las muestras fueron compactadas bajo el mismo contenido inicial de agua y diferente densidad seca, éstas siguen una trayectoria similar. Estos resultados se presentan en las figuras 4,6 y 8 .

Las curvas exploratorias mostradas en las figuras 4 y 6 no parten de una saturación correspondiente al $100 \%$ puesto que las muestras fueron compactadas a densidades y contenidos de agua que se encontraban por debajo del contenido de agua de saturación. Por el contrario, las curvas exploratorias mostradas en la figura 8 corresponden a muestras que fueron compactadas bajo un contenido de agua cercano al contenido de agua de saturación, por lo que presentan una trayectoria de secado más completa. De esta manera, en ninguno de los casos el suelo parte de una succión correspondiente a $0 \mathrm{kPa}$, por lo que en el caso de la muestra 1 a la muestra 4 no se está obteniendo una representación de los poros más grandes, mientras que las muestras $5 \mathrm{y}$ 6 presentan la contribución de un mayor rango de tamaño de poros.

La fig. 3 muestra la distribución del tamaño de poros para las curvas exploratorias de las muestras 1 y 2 . Puede apreciarse que la relativa homogeneidad que presentan las curvas exploratorias se ve reflejada en una función de distribución de tamaño de poros semejante mientras que el tamaño de poros promedio predominante para ambas curvas está marcado por un valor cercano a los 20.000 Angstrom $(2 \mu \mathrm{m})$, teniendo en cuenta que las muestras fueron compactadas bajo diferentes densidades secas iniciales pero el mismo contenido de agua.

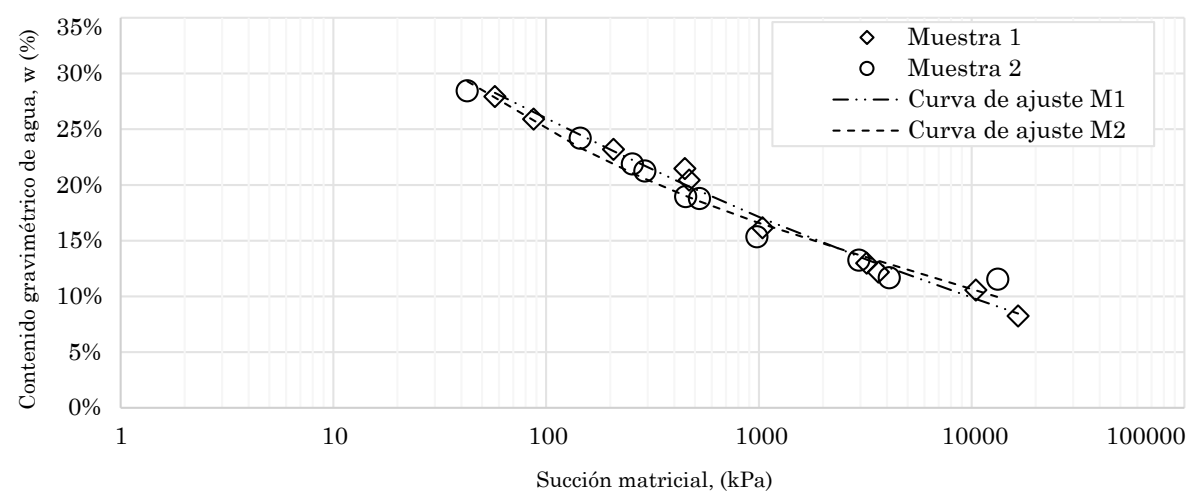

Fig. 2. Curvas exploratorias de secado. Muestras 1 y 2. Fuente: Autores

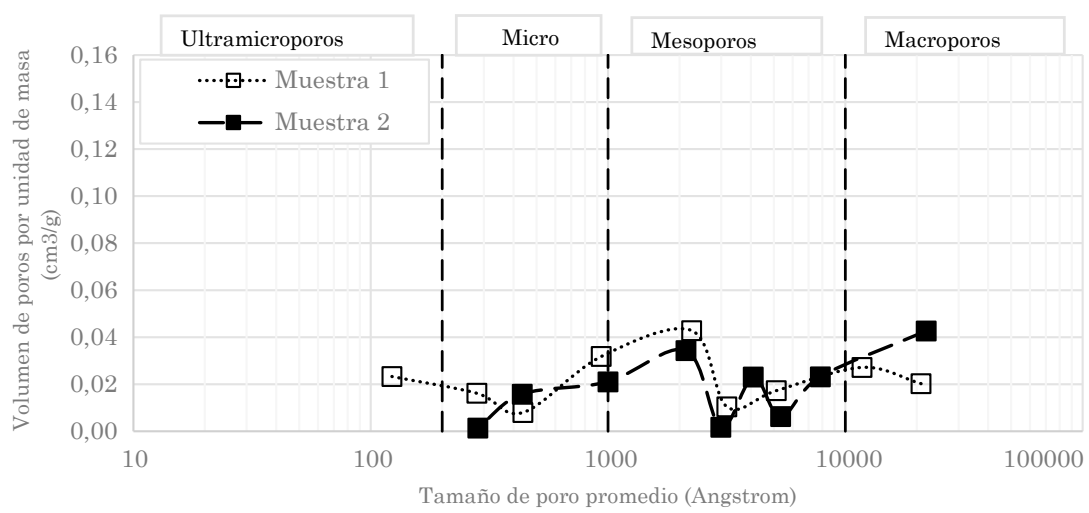

Fig. 3. Función de distribución del tamaño de poros para un suelo compactado bajo densidades de $0.95 \mathrm{~g} / \mathrm{cm} 3 \mathrm{y} 1.15 \mathrm{~g} / \mathrm{cm} 3 \mathrm{y}$ un contenido de agua de $28 \%$.

Fuente: Autores 


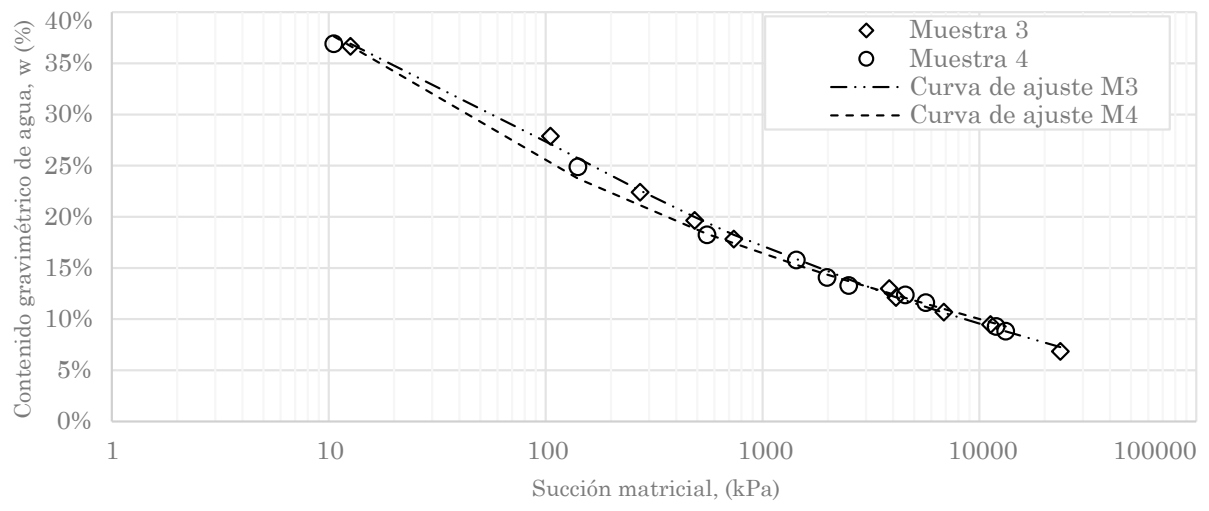

Fig. 4. Curva exploratoria de secado. Muestras 3 y 4 .

Fuente: Autores

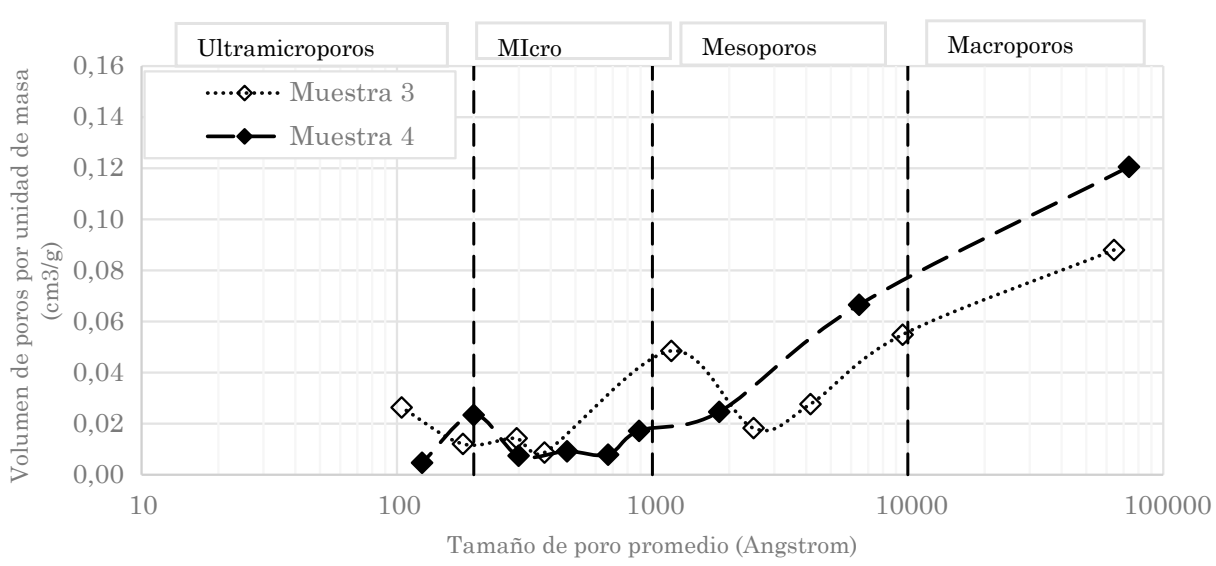

Fig. 5. Función de distribución del tamaño de poros para un suelo compactado bajo densidades de $0.95 \mathrm{~g} / \mathrm{cm} 3$ y $1.15 \mathrm{~g} / \mathrm{cm} 3$ y un contenido de agua de $38 \%$.

Fuente: Autores

La fig. 4 muestra que en un rango de succión comprendido entre los $100 \mathrm{kPa}$ y los $500 \mathrm{kPa}$, la curva exploratoria de secado para la muestra 3 , cuya densidad seca es de $1,15 \mathrm{~g} / \mathrm{cm}^{3}$, se encuentra levemente a la derecha de la curva exploratoria de la muestra 4, compactada a una densidad seca de $0,95 \mathrm{~g} / \mathrm{cm}^{3}$. Los mayores valores de succión para la muestra 3 estarían relacionados con una estructura más cerrada asociada a una densidad seca de compactación más alta, en la se genera una mayor capacidad de almacenamiento de agua y, por tanto, se convierte en una estructura más impermeable.

Ese hecho se refleja en el comportamiento de la función de distribución de tamaño de poro que se muestra en la fig. 5, en donde la muestra 3 se halla por debajo de la muestra 4 para tamaños de poro entre 2000 Angstrom $(0.2 \mu \mathrm{m})$ y 70000 Angstrom (7 $\mu \mathrm{m})$.

Hacia los $4000 \mathrm{kPa}$, la curva exploratoria de la muestra 4 se encuentra a la derecha de la muestra 3 , lo que se ve reflejado en un pico distintivo en la función de distribución del tamaño de poro. El tamaño de poros promedio predominante para ambas curvas está marcado por un valor cercano a los 80000 Angstrom ( $8 \mu \mathrm{m})$.

En la fig. 6, se muestra que en un rango de succión comprendido entre los $50 \mathrm{kPa}$ y los $700 \mathrm{kPa}$, la curva exploratoria de secado para la muestra 6 se encuentra levemente a la derecha de la curva exploratoria de la muestra 5. En ese rango de succiones no existe un mayor número de puntos que puedan confirmar este comportamiento, aunque parece existir una tendencia homogénea entre las dos curvas exploratorias analizadas.

El comportamiento atípico de la muestra 6, asociado con los datos obtenidos para la modelación de la curva, se ve reflejado en la fig. 6 , en donde se observa un pico distintivo dentro de la función de distribución de poros para dicha muestra hacia los 20000 Angstrom $(2 \mu \mathrm{m})$. El tamaño de poros promedio predominante para ambas curvas está marcado por un valor cercano a los 200000 Angstrom (0.02 mm). 


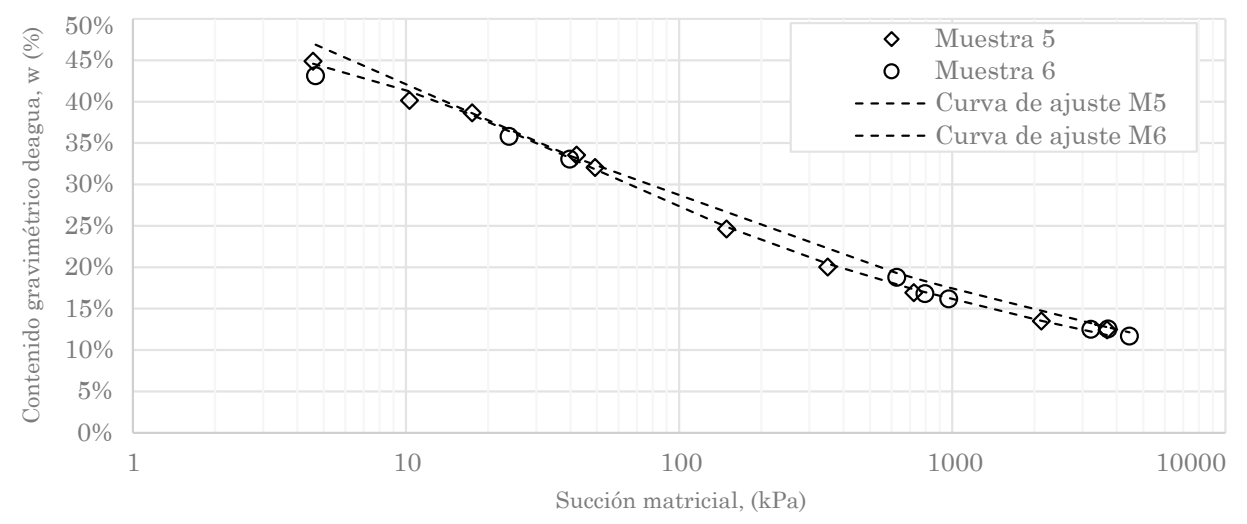

Fig. 6. Curvas exploratorias de secado. Muestras 5 y 6.

Fuente: Autores

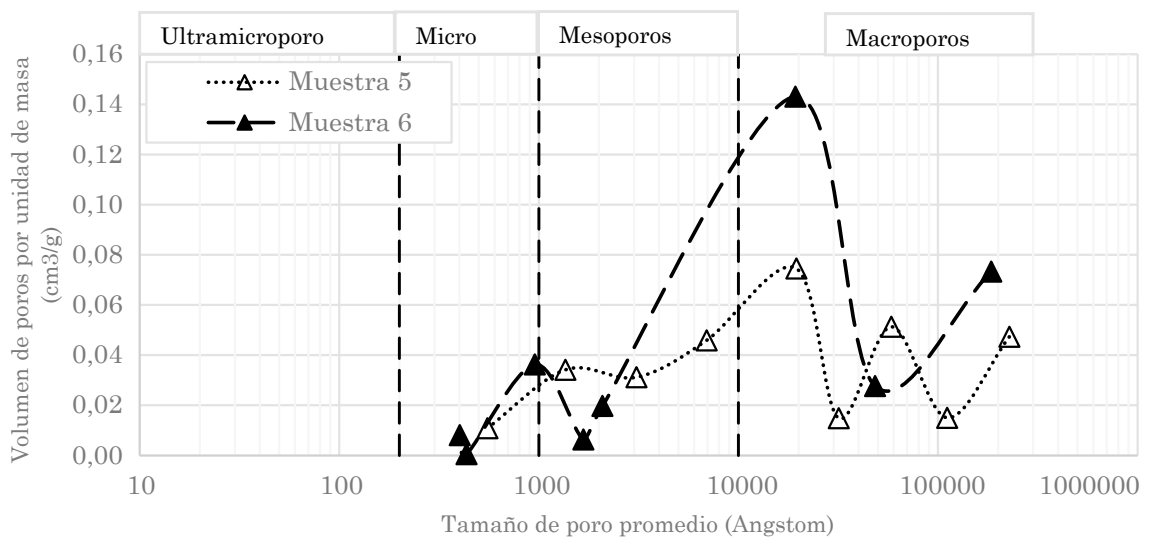

Fig. 7. Función de distribución del tamaño de poros para un suelo compactado bajo densidades de $0.95 \mathrm{~g} / \mathrm{cm} 3$ y $1.15 \mathrm{~g} / \mathrm{cm} 3$ y un contenido de agua de $45 \%$ Fuente: Autores

De acuerdo con los resultados obtenidos, se aprecia que la densidad seca inicial del material no influye en las trayectorias que describen las curvas exploratorias para valores similares de succión. Al parecer, la curva exploratoria es controlada únicamente por el contenido de agua de compactación, sin que la densidad seca inicial tenga influencia en su comportamiento.

Este comportamiento se ve reflejado en el hecho de que, para los tres grupos de muestras analizados, el tamaño de poros promedio predominante para un contenido de agua en específico fue el mismo y su variación se presentó en la medida en que el contenido de agua de compactación de cada uno de los grupos analizados aumentó. Por otro lado, los puntos iniciales de las curvas exploratorias mostradas en las figuras 4, 6 y 8 representan el estado de succión-contenido de agua del material compactado bajo las características de compactación definidas en la Fig. 3. Se encontró que, para muestras compactadas del lado seco del contenido de agua óptimo y cercano al óptimo bajo diferente densidad seca, la succión aumenta al disminuir el contenido de agua de compactación, presentándose un aspecto casi vertical de las curvas iso-succión. Como es de esperarse, altos valores de contenido de agua generan bajos valores de succión, sin embargo, éstos no presentan una tendencia paralela a las líneas de igual saturación, contrario a lo encontrado por [3], lo que se podría atribuir a que para bajos valores de densidad seca de compactación, la succión es controlada por el contenido de agua, sin que se presente influencia de la densidad en la succión. Posiblemente, a mayores valores de densidad seca de compactación pueda ponerse en evidencia un cambio en este comportamiento, sin embargo, se carece de la información suficiente para confirmar este comportamiento.

\section{B. Influencia del contenido de agua}

En la figura 8 y la figura 9 puede observarse la relación entre el contenido gravimétrico de agua y la succión de muestras compactadas bajo dos densidades secas: $0,95 \mathrm{~g} / \mathrm{cm}^{3}$ y $1,15 \mathrm{~g} / \mathrm{cm}^{3}$, respectivamente. El contenido inicial de agua seleccionado para el presente estudio representa la condición seca, la condi- 
ción óptima y la condición húmeda del material estudiado. Al igual que en el caso anterior, los resultados se analizaron a través de la comparación del contenido gravimétrico de agua con la succión matricial.

Se observa en ambos casos que las curvas exploratorias para las muestras compactadas bajo la misma densidad con un contenido de agua de compactación del lado húmedo del óptimo se ubican del lado derecho de la gráfica. En el otro sentido, las curvas exploratorias para las muestras compactadas bajo un contenido de agua del lado seco del óptimo tienden a ubicarse del lado izquierdo de la curva. El contenido de agua de compactación correspondiente al óptimo se encuentra entre las dos curvas.

Este comportamiento podría atribuirse a la estructura que presenta el material al ser compactado bajo diferentes contenidos de agua. De acuerdo con [18], un suelo compactado del lado seco del óptimo presentará una fábrica abierta con poros relativamente grandes e interconectados. Los espacios de poros del mismo suelo compactado a un contenido de agua del lado húmedo del óptimo no están generalmente interconectados o están en un estado cerrado. Estos suelos tienen una alta capacidad de retención de agua debido a que presentan una estructura más cerrada y no es posible apreciar la interconexión entre partículas, lo que los hace más resistentes al secado. La frontera entre un espacio de poros cerrado y las condiciones de poro abiertas ocurre aproximadamente cerca del contenido óptimo de agua.

Para el caso de la fig. 8, se observa que cuando las muestras analizadas alcanzan una succión de 800 $\mathrm{kPa}$, las trayectorias de secado tienden a seguir una relación de secado común, mientras que para el caso de la figura 9, la relación de secado común se presenta sobre los $3000 \mathrm{kPa}$. Esto sugiere que a partir de estos valores de succión las trayectorias de secado no dependen del contenido de agua de compactación o la densidad seca inicial.

\section{Influencia del secado inicial del material previo a la compactación}

La referencia [3] desarrolló un programa experimental para conocer las propiedades específicas de un suelo derivado de ceniza volcánica, correspondiente al estrato más superficial de una zona representativa del suelo típico del área de Palestina, Caldas, Colombia, teniendo en cuenta algunas recomenda-

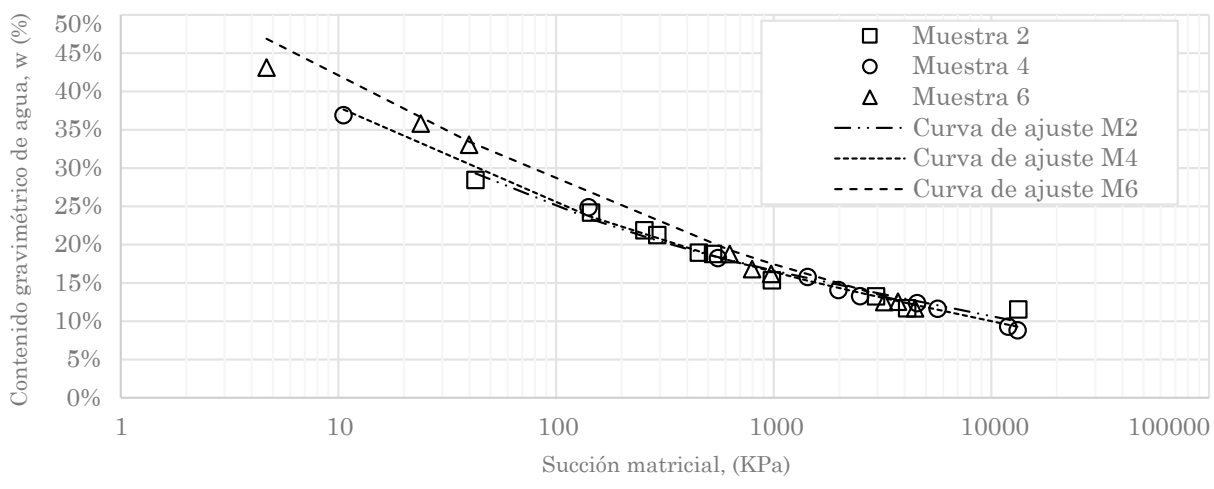

Fig. 8. Curvas exploratorias de secado. Muestras 2, 4 y 6 . Fuente: Autores

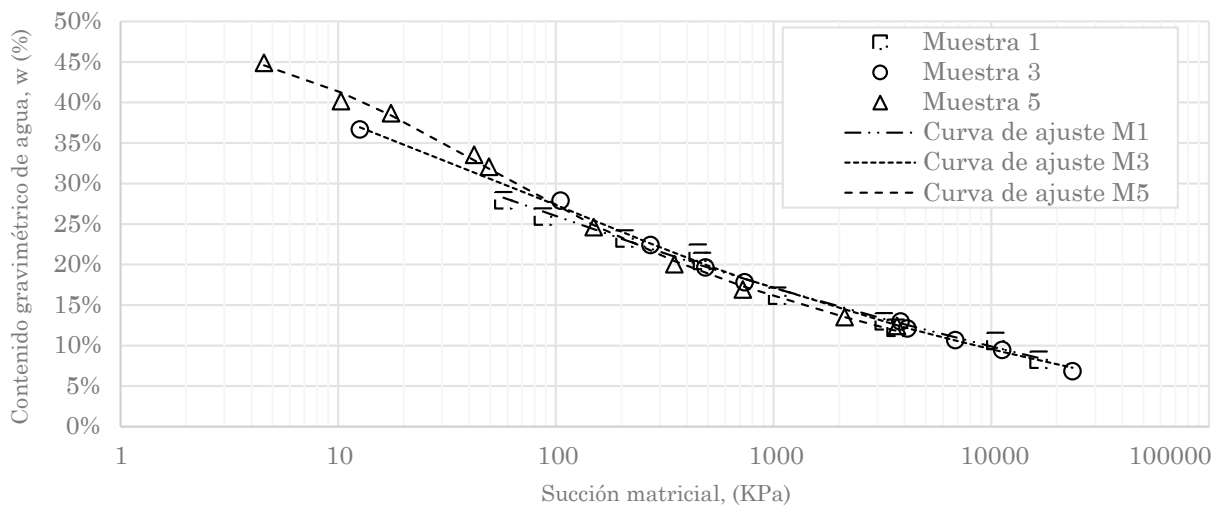

Fig. 9. Curvas exploratorias de secado. Muestras 1, 3 y 5. Fuente: Autores 
ciones particulares para la caracterización de suelos residuales tropicales. La Tabla 4 muestra la caracterización básica del material analizado.

La fig. 10 muestra la curva exploratoria para la muestra 6 de este estudio, compactada bajo una densidad seca de $0,95 \mathrm{~g} / \mathrm{cm}^{3}$ y un contenido de agua de $45 \%$, junto con la curva exploratoria del mismo suelo analizado por [3] y compactada bajo una densidad de $0,95 \mathrm{~g} / \mathrm{cm}^{3}$ y un contenido de agua de $56,3 \%$, que pretendía simular las condiciones encontradas para el material natural.

Los resultados encontrados por [3] para un tipo de suelo limo areno arcilloso, como fue descrito inicialmente, y analizado en estado compactado difieren de los presentados anteriormente. Al parecer, los procesos de secado modifican algunas propiedades del suelo, en especial la plasticidad. Además, el tiempo y las condiciones iniciales de almacenamiento, la mineralogía y cambios en los procesos químicos y físicos asociados con estos factores están generando cambios de propiedades en suelos de este tipo.
Se sabe que los materiales derivados de ceniza volcánica compuestos por alófana y haloisita pierden sus características de plasticidad cuando son secados al aire o en horno. Probablemente este factor esté estrechamente ligado con el cambio en las propiedades iniciales del suelo encontradas en este estudio (p.e. contenido inicial de agua, límites de consistencia, gravedad específica, granulometría) en comparación con las halladas por [3]. De esta manera, la pérdida de la plasticidad, así como el cambio en otras propiedades del suelo, podría estar relacionado con el desplazamiento de la curva exploratoria de la muestra 6 hacia la izquierda, en comparación con la curva exploratoria de la muestra limo areno arcillosa encontrada por [3]. Es deseable estudiar los cambios en la estructura mineralógica del suelo asociados a un proceso de secado al aire y su efecto sobre la forma que describe la trayectoria seguida por la curva exploratoria, así como la influencia que tendrían minerales como la alófana y la haloisita en la compactación del suelo bajo esta condición.

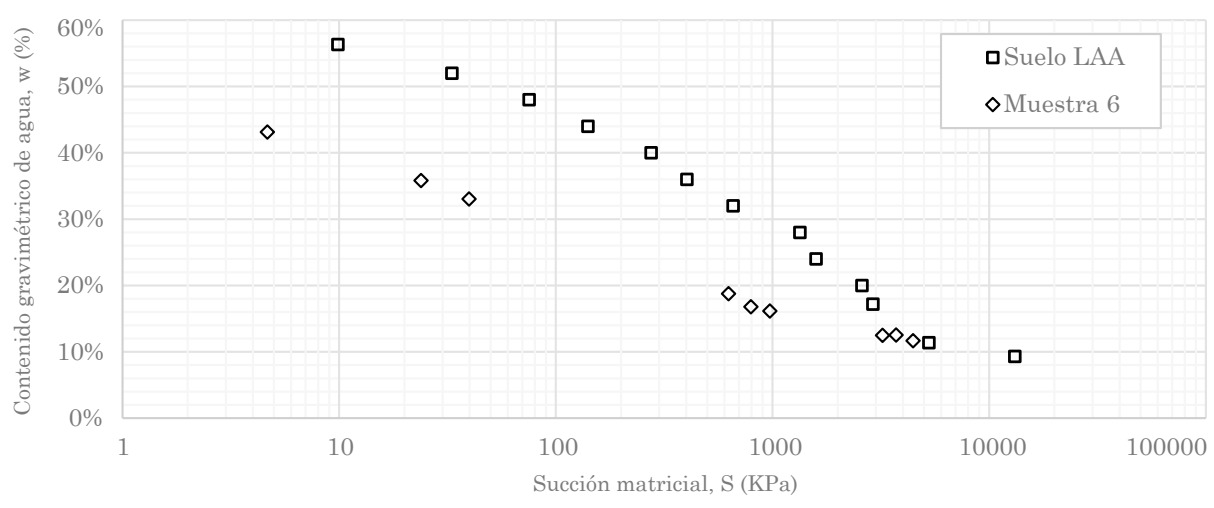

Fig. 10. Curvas exploratorias para suelos derivados de ceniza volcánica. Fuente: Autores.

Tabla 4. Características generales del suelo estudiado por [3].

\begin{tabular}{|c|c|}
\hline \multicolumn{2}{|c|}{ Características del suelo antes de secado } \\
\hline Descripción visual & $\begin{array}{c}\text { Limo areno arcilloso carmelito pálido medio con vetas } \\
\text { grises y presencia de oxidación en tonos rojizos }\end{array}$ \\
\hline Color & Carmelito pálido \\
\hline Contenido de agua natural (\%) & 58,0 \\
\hline Gravedad específica & 2,63 \\
\hline Límite líquido (\%) & 58,7 \\
\hline Límite plástico (\%) & 37,0 \\
\hline Índice de plasticidad (\%) & 21,7 \\
\hline Partículas de tamaño arena (\%) & 42,75 \\
\hline Partículas de tamaño limo (\%) & 53,91 \\
\hline Partículas de tamaño arcilla (\%) & 3,34 \\
\hline Clasificación USCS & $\mathrm{MH}$ \\
\hline
\end{tabular}

Fuente: datos tomados de [3] 
Finalmente, las curvas exploratorias analizadas permiten estimar las condiciones de succión de un material seco al aire como el que se podría encontrar en la zona de estudio, lo que ayudará a abordar la implementación de la mecánica de suelos parcialmente saturados en la solución adecuada de problemas de ingeniería geotécnica asociados a la estabilidad de taludes, el análisis del flujo de agua y la conductividad hidráulica o la predicción de comportamientos expansivos o colapsables del suelo sometido a condiciones de carga variables. Se sabe que la succión contribuye en el aumento de la resistencia al corte del suelo, por lo que es común ver en este tipo de suelos derivados de ceniza volcánica cortes de talud verticales asociados a altos valores de succión. Altos valores de succión estarían también asociados a una reducción de la conductividad hidráulica como producto de una fábrica más cerrada que reduce la probabilidad de que el agua en estado líquido fluya a través de los poros llenos de agua y afecte las capas inferiores de suelo, lo que permite protegerlas del efecto adverso de la erosión. En una muestra de suelo compuesta por tres fases (sólida, líquida y gaseosa), cuando se ha alcanzado un estado residual en la fase líquida, ésta pierde su capacidad de moverse como una fase enlazada, convirtiéndose en una fase aparentemente desconectada. Este estado se obtiene cuando la película de agua se vuelve tan delgada y, por lo tanto, fuertemente adsorbida por la fase sólida, que pierde su capacidad de responder a los gradientes hidráulicos causando una disminución en la conductividad hidráulica [11]hysteretic unsaturated fluid phase content (retention.

De acuerdo con lo observado anteriormente, las trayectorias que describen las curvas exploratorias compactadas bajo la condición húmeda de contenido de agua óptimo son las que mejor se comportarán en el análisis de los problemas de ingeniería geotécnica, puesto que, bajo esta condición de compactación, fueron las que presentaron mayores valores de succión. Así, para la estabilidad de taludes, por ejemplo, en la medida en que la capa expuesta al medio ambiente sea capaz de controlar la permeabilidad, se protegerá el talud ante posibles deslizamientos causados por la infiltración de agua, además de generarse una mayor resistencia al corte asociada a las altas succiones.

\section{Conclusiones}

Los resultados del estudio realizado al material permiten formular las siguientes conclusiones:

Se observó que en las curvas exploratorias de secado, compactadas bajo dos densidades secas diferentes y el mismo contenido de agua, la succión es independiente de la densidad seca inicial del material. Además de que la succión aumenta al disminuir el contenido de agua de compactación.

En los tres casos analizados se observó que las curvas exploratorias siguen una trayectoria común, por lo que parece ser que la succión es controlada únicamente por el contenido de agua, sin que la densidad seca inicial tenga influencia en su comportamiento.

Este comportamiento se vio reflejado en el hecho de que, para los tres casos analizados, el tamaño de poros promedio predominante para un contenido de agua específico fue el mismo y su variación se presentó en la medida en que el contenido de agua de compactación de cada uno de los casos analizados aumentó.

Para muestras compactadas del lado seco del contenido óptimo de agua, las curvas exploratorias se localizan a la izquierda de las de muestras compactadas del lado húmedo del óptimo. En el caso de muestras compactadas bajo un contenido de agua óptimo, las curvas exploratorias se ubican en el medio de las dos anteriormente descritas. Este comportamiento puede atribuirse a la fábrica que presenta el material al ser compactado bajo diferentes contenidos de agua. Un suelo compactado del lado seco del óptimo presentará una fábrica abierta con poros relativamente grandes e interconectados. Para un suelo compactado del lado húmedo del óptimo, los poros no están interconectados y su fábrica es mucho más cerrada, lo que les da una mayor capacidad de retención de agua.

En el caso en que se analizaron las muestras compactadas bajo la misma densidad seca y diferente contenido de agua, para valores de succión mayores a $800 \mathrm{kPa}$ asociados a una densidad seca de compactación de $0,95 \mathrm{~g} / \mathrm{cm}^{3}$, las trayectorias de secado tendieron a seguir una relación común. En el caso de muestras compactadas bajo una densidad de 1,15 g/ $\mathrm{cm}^{3}$, las trayectorias de secado tendieron a una curva común hacia los $3000 \mathrm{kPa}$. A partir de estos valores de succión parece ser que las trayectorias de secado no dependen del contenido de agua de compactación o la densidad seca inicial.

El suelo analizado presentó un fuerte cambio en sus propiedades índice como resultado del secado inicial. Al parecer, los procesos de secado modifican algunas propiedades del suelo como la plasticidad. El suelo seco no presenta la capacidad de retención de agua inicial y no permite la formación de una estructura parecida a la desarrollada por un suelo con un contenido de agua natural.

Las curvas exploratorias analizadas permiten estimar las condiciones de succión de un material seco al aire, como el que se podría encontrar en la zona de estudio, lo que permite estimar parámetros de resistencia del material bajo condiciones similares a las establecidas en laboratorio.

\section{Agradecimientos}

Este trabajo se desarrolló dentro del marco del Programa Nacional de Semilleros de Investigación, Creación e Innovación, de la Universidad Nacional 
de Colombia 2013-2015 - V Corte. Por lo anterior, los autores expresan su agradecimiento a la Vicedecanatura de Extensión e Investigación de la Facultad de Ingeniería y a la Universidad Nacional de Colombia por el apoyo brindado en esta investigación.

\section{Financiación}

Artículo de Investigación derivado del proyecto de investigación titulado: "Análisis del comportamiento volumétrico de suelos residuales, sometidos a ciclos de humedecimiento-secado". Código del proyecto: 28889 Código Quipú: 201010023525. Financiado por la Dirección de Investigación de la Universidad Nacional de Colombia, Sede Bogotá. Fecha de inicio: 13 de mayo de 2015. Fecha de finalización: 13 de noviembre de 2015 .

\section{Referencias}

[1] A. Lizcano, M. C. Herrera y J. C. Santamarina, "Suelos derivados de ceizas volcánicas en Colombia," Rev. Int. Desastr. Nat. Accid. e Infraestruct. Civ., vol. 6, no. 2, pp. 167-197, 2006.

[2] D. G. Fredlund, "The implementation of unsaturated soil mechanics into geotechnical engineering," Can. Geotech. J., vol. 37, pp. 963-986, 2000. https://doi.org/10.1139/t00-026

[3] L. Viveros, "Influencia del proceso de compactación en la re sistencia al corte de un suelo derivado de ceniza volcánica," Tesis de maestría, Universidad Nacional de Colombia, Bogotá, Colombia, 2014.

[4] R. J. Chandler, M. S. Crilly y G. Montgomery-Smith, "A low cost method for assessing clay desiccation for low-rise buildings," Proc. ICE, vol. 92, no. 2, pp. 82-89, 1992. https://doi. org/10.1680/icien.1992.18771

[5] D. G. Fredlund y A. Xing, "Equations for the soil-water characteristic curve," Can. Geotech. J., vol. 31, no. 6, pp. 1026 1026, 1994. https://doi.org/10.1139/t94-120

[6] N. Lu y W. J. Likos, Unsaturated soil mechanics. New York: Wiley, 2004

[7] O. A. Chadwick, R. T. Gavenda, E. F. Kelly, K. Ziegler, C. G. Olson, W. C. Elliott y D. M. Hendricks, "The impact of climate on the biogeochemical functionung of volcanic soils," Chem. Geol., vol. 202, pp. 195-223, 2003. https://doi. org/10.1016/j.chemgeo.2002.09.001
[8] F. C. Townsed, "Geotechnical characteristisd of residual soils," J. Geotech. Eng., vol. 11, no. 1, pp. 77-94, 1985. https://doi.org/10.1061/(ASCE)0733-9410(1985)111:1(77)

[9] L. Wesley, "Behaviour ang geotechnical properties of residual soils and allophane clays," Obras y Proy., vol. 6, pp. $5-10,2009$.

[10] J. A. Capdevila, "Comportamiento Tensión-Deformación del Loess del Centro de Argentina en Campo y Laboratorio: Influencia de los Parámetros Estructurales.," Universidad Nacional de Cordoba, 2008.

[11] L. Luckner, M. T. Van Genuchten, and D. R. Nielsen, "A consistent set of parametric models for the two phase flow of immiscible fluids in the subsurface," Water Resour. Res., vol. 25, no. 10, pp. 2187-2193, 1989. https://doi. org/10.1029/WR025i010p02187

[12] A. Gens, E. E. Alonso, J. Suriol, and A. Lloret, "Effect over structure on the volumetric behaviour of compacted soil," in Unsaturated Soils, Balkema, 1995, pp. 83-88.

[13] L. Wesley, "Geotechnical characterization and behavior of allophane clays," Proc. Int. Wirkshop Characterisation Eng. Prop. Nat. Soils, vol. 2, pp. 1379-1399, 2003.

[14] ASTM D698 - 12e1, "Standard Test Methods for Laboratory Compaction Characteristics of Soil Using Standard Effort (12 $400 \mathrm{ft}-\mathrm{lbf} / \mathrm{ft} 3(600 \mathrm{kN}-\mathrm{m} / \mathrm{m} 3))$," in 2012 Annual Book of ASTM Stanards, Philadelphia: ASTM International, 2012.

[15] ASTMD1557, "Standard Test Methods for Laboratory Compaction Characteristics of Soil Using Modified Effort $(56,000 \mathrm{ft}-\mathrm{lbf} / \mathrm{ft} 3(2,700 \mathrm{kN}-\mathrm{m} / \mathrm{m} 3))$, , in 2012 Annual book of ASTM Standards, Philadelphia: ASTM International, 2012.

[16] J. K. S. Venkatarama B.V., "The static compaction of soils Technical Note," Géotechnique, vol. 43, no. 2, pp. 337-341, 1993. https://doi.org/10.1680/geot.1993.43.2.337

[17] J. C. García, "Efectos de los cambios de humedad en la resistencia de un suelo parcialmente saturado derivado de ceniza volcánica," Universidad Nacional de Colombia, 2003.

[18] S. K. Vanapalli, D. G. Fredlund y D. E. Pufahl, "The influence of soil structure and stress history on the soilwater characteristics of a compacted till," Geotechnique, vol. 49 , no. 2 , pp. $143-159,1999$. https://doi.org/10.1680/ geot.1999.49.2.143

[19] N. Perez García, "Determinación de las curvas caracterís ticas en suelos no saturados con celdas de presión," Natl. Acad. Sci., no. 313, p. 54, 2008. 